\title{
ORIGINAL
}

\section{EL PROYECTO EPICARDIAN: UN ESTUDIO DE COHORTES SOBRE ENFERMEDADES Y FACTORES DE RIESGO CARDIOVASCULAR EN ANCIANOS ESPAÑOLES: CONSIDERACIONES METODOLÓGICAS Y PRINCIPALES HALLAZGOS DEMOGRÁFICOS}

Rafael Gabriel Sánchez (1), Blanca Novella Arribas (2), Margarita Alonso Arroyo (1), Saturio Vega Quiroga (3), Isidro López García (4), Carmen Suárez Fernández (1) y Javier Muñiz García (5)

(1) Unidad de Epidemiología Clínica e Investigación. Hospital La Princesa. Madrid

(2) Centro de Salud Potosí. Area II de Salud. Madrid

(3) Centro de Salud de Arévalo. Avila

(4) Centro de Salud de Begonte. Lugo

(5) Instituto Universitario de Ciencias de la Salud. Hospital Juan Canalejo. Universidad de La Coruña

(*) Este proyecto ha sido financiado por el Fondo de Investigación Sanitaria (Expediente FIS 93/012 y FIS 96/1993 y por DGICyT: PB 1255-Co4.

\section{RESUMEN}

Fundamentos: A pesar de que la incidencia de cardiopatía isquémica es más alta en sujetos mayores de 65 años, la mayor parte de la investigación cardiovascular se ha centrado en la población adulta de mediana edad, careciendo en España hasta la fecha de un estudio de cohortes en este grupo de población. El objetivo de este trabajo es revisar el papel y la metodología de los estudios de cohortes como herramienta epidemiológica imprescindible para la investigación de la prevalencia e incidencia de angina, IAM, ictus, y sus principales factores de riesgo cardiovascular.

Métodos: Estudio de cohortes en tres zonas de España: Madrid (barrio de Lista), Arévalo (Avila) y Begonte (Lugo). Muestra aleatoria estratificada por edad y sexo a partir de los padrones municipales de cada zona y municipio $(n=5,079)$. Evaluación basal de la cohorte en dos fases: domiciliaria mediante encuesta estructurada para cribado de enfermedad isquémica cardiaca y factores de riesgo clásicos (hipertensión, dislipemia, diabetes y tabaquismo) y clínica para confirmación de casos. En la fase de seguimiento se utilizó el «método de búsqueda en frío» del proyecto MONICA modificado para la búsqueda e indagación de casos incidentes usando todos los informes clínicos hospitalarios y de atención primaria para la confirmación del evento cardiovascular. Adicionalmente se solicitó al INE información sobre causa y fecha de la muerte de los individuos de la cohorte fallecidos.

Correspondencia:

Rafael Gabriel Sánchez

Unidad de Investigación.

Epidemiología Clínica

Hospital La Paz

Paseo de la Catellana, 261

28046 Madrid

Correo electrónico: rgabriel.hulp@salud.madrid.org

\section{ABSTRACT}

The EPICARDIAN Project: a Cohort Study on Cardiovascular Diseases and Risk Factors among the Elderly in Spain: Methodological Aspects and Major Demographic Findings

Background: Despite a greater incidence of ischemic heart disease among individuals over age 65 , most cardiovascular research has been focused on the middle-aged adult population. To date no cohort study on this population have been made in Spain. This study is aimed as reviewing the role and methodology of cohort studies as an epidemiological tool absolutely essential for researching the prevalence and incidence of angina, AMI, stroke and the major cardiovascular risk factors.

Methods: Cohort study in three areas of Spain (Lista district in Madrid), Arevalo (Avila) and Begonte (Lugo).Age and sex stratified random sample by based on the municipal censuses of each area and municipality $(\mathrm{n}=5,079)$. Two-stage initial cohort assessment: home survey structured for the screening ischemic heart disease and classic risk factors (hypertension, dyslipemia, diabetes and smoking habit) and clinical assesment for case confirmation. In the follow-up phase the MONICA project «cold pursuit» method modified for pinpointing and investigating indicent cases was used, employing all of the hospital and primary care clinical records for confirming the cardiovascular event. Data was also requested from the Spanish National Institute of Statistics as to the cause and date of death of the deceased individuals in the cohort. 
Resultados: La prevalencia global de IAM seguro fue del $4 \%$ (IC 95\%: $3,4 \%$ y $4,5 \%$ ), y de IAM seguro más probables del $6,2 \%$ (IC $95 \%: 5,5-6,9)$. La prevalencia de IAM seguro fue más elevada en los varones $6,7 \%$ (IC 95\%:5,63-7,79) que en las mujeres, $2 \%$ (IC $95 \%: 1,51-2,55)(\mathrm{p}<0,001)$. La prevalencia de hipertensión según criterios JNCVI fue $68 \%$, de hipercolesterolemia $26,4 \%$ según criterios del NCEP, de diabetes $13,4 \%$ según criterios de la OMS y el 11,3\% eran fumadores. La incidencia acumulada en 3,2 años de IAM seguro no fat fue del 1,4\% (IC 95\%: 1,1-1,8); 1,1\% (IC95\%:0,74 1,37) de (IC95\%: 0,824-1,48) de IAM te por IAM y $1,13 \%$ (IC $95 \%$ : 0,824-1,48) de muerte súbita.

Conclusiones: La población de ancianos incluida en este estudio presenta una elevada prevalencia de factores de riego cardiovascular, así como tasas de incidencia de cardiopatía isquémica tres veces superiores a las de la población adulta española de mediana edad. En las mujeres, el perfil de riesgo es significativamente peor que en los hombres, lo que puede ser debido a la mayor mortalidad en edades más precoces en el sexo masculino.

Palabras clave: Estudios de cohortes. Cardiopatía isquémica. Incidencia. Personas mayores.

\section{INTRODUCCIÓN}

La cardiopatía isquémica es una de las enfermedades crónicas más relevantes, tanto en términos de mortalidad como de años de vida perdidos ajustados por calidad de vida ${ }^{1-3}$. La mortalidad por cardiopatía isquémica ha experimentado un descenso promedio de un $16 \%$, En el momento actual, la mayor incidencia de esta enfermedad se observa en el segmento etario de más edad, suponiendo la primera causa de mortalidad en mayores de 65 años (15\% de la mortalidad total), y la segunda de deterioro y pérdida de independencia tras el ictus. En el año 2000 se produjeron en España cerca de 39.000 defunciones por enfermedad isquémica del corazón que supusieron el $31 \%$ del total de defunciones de origen cardiovascular (40\% para los hombres y $30 \%$ para las mujeres). Aunque las tasas son más altas a partir de los 65 años ${ }^{6}$ la mayor parte de la investigación cardiovascular se ha centrado en la población adulta de mediana edad, siendo escasa la representación del colectivo de ancianos en los estudios realizados hasta la fecha ${ }^{7}$. Si se pretende aumentar la supervivencia y mejorar la calidad de vida, es imprescindible el conocimiento de la patogenia de la enfermedad, de su historia natural, así como de sus determinantes en este grupo de edad, siendo aquí donde un estudio de cohortes resulta pertinente en este grupo de población.
Results: The overall AMI prevalence was 4\% (95\% CI: 3.4\%, 4.5\%); definite plus probable AMI being 6.2\% (95\% CI: 5.5-6.9) The definite AMI prevalence was higher among the men $6.7 \%(95 \%$ CI: 5.63-7.79) than among the women, $2 \%$ (95\% CI: 1.51-2.55) (p< $0.001)$. Hypertension prevalence according to JNCVI criteria was $68 \%$, hypercholesterolemia $26.4 \%$ according to NCEP criteria, diabetes prevalence $13.4 \%$ according to $\mathrm{WHO}$ criteria, and $11.3 \%$ were smokers. The cumulative incidence for a 3.2-year period for nonfatal definite AMI was $1.4 \%$ (95\% CI: 1.1-1.8); $1.1 \%$ (95\% CI: 0.74 1.37) proble AMI; 1.17 (IC95\%: 0.824-1.48) for fat C:0.74AMI or death due to AMI and 1.13\% (IC 95\%: 0.824-1.48) for sudden death.

Conclusions: The elderly population included in this study shows a high prevalence of cardiovascular risk factors, as well as ischemic heart disease incidence rates three times higher than those of the middle-aged adult population in Spain. The risk profile for higher death rate at earlier ages among men.

Key words:Aging. Cohort studies. Cardiovascular diseases. Spain. Incidence.

A partir de la década de los 50 se iniciaron numerosos estudios epidemiológicos de cohortes sobre enfermedad cardiovascular, cuyos hallazgos han permitido conocer tanto su distribución, como comprender mejor su etiología. En población adulta de mediana edad destacan entre otros el Estudio de Framingham, el «Pooling Project» y el Estudio de los Siete Países y en ancianos el Honolulu Heart Project y el Cardiovascular Health Study $y^{8-12}$. En España hasta la fecha solo existía el estudio prospectivo de los Trabajadores de Manresa ${ }^{13}$, cuyo diseño y resultados se muestran en otro articulo de esta monografía ${ }^{14}$.

Se pretende en este articulo revisar los estudios de cohortes como herramienta epidemiológica imprescindible para la investigación cardiovascular, utilizando como ejemplo el Estudio EPICARDIAN, en el que se estudian la prevalencia e incidencia de angina, IAM, ictus, y los principales factores de riesgo cardiovascular: hipertensión, dislipemia, diabetes y tabaquismo ${ }^{15}$.

\section{MATERIAL Y METODOS}

El objetivo principal de un estudio de cohortes es determinar la aparición de nuevos casos de enfermedad en grupos de individuos expuestos a diferentes niveles de dis- 
tintos factores de riesgo con los que presumiblemente están relacionados ${ }^{16}$. El proyecto EPICARDIAN pretende estimar la incidencia de las enfermedades cardiovasculares en población anciana española y estimar el riesgo de padecer la enfermedad que tienen los individuos expuestos a los diferentes factores de riesgo cardiovascular conocidos frente a los no expuestos ${ }^{15}$.

Los estudios de cohortes pueden ser prospectivos (concurrentes), retrospectivos (históricos) o mixtos, dependiendo de la relación temporal entre la iniciación del estudio y la ocurrencia de la enfermedad. El estudio que nos ocupa es prospectivo, pues el nivel de exposición se define al principio del estudio y se observa la ocurrencia subsecuente de la enfermedad mientras el estudio se desarro1la. Esto ha permitido determinar a priori (antes de iniciar la investigación) los criterios de medida de la exposición y recoger la información sobre la exposición al inicio del seguimiento (en condiciones basales).

En general, cuando la exposición y la enfermedad son muy frecuentes, como es el caso de la patología cardiovascular, los estudios de cohortes se realizan en una población de un área geográfica determinada en la que se asegura la variación adecuada de la exposición en la población y de la incidencia de la enfermedad. En el estudio EPICARDIAN se seleccionó una muestra de la población mayor de 65 años de 4 áreas de salud (Barrio de Lista en Madrid, Las Margaritas en Getafe, la comarca de Arévalo en Ávila y la de Begonte en Lugo). Estas zonas fueron elegidas debido principalmente a la accesibilidad y a las posibilidades de seguimiento de su población, circunstancias imprescindibles en un estudio de cohortes.

Las poblaciones elegidas en el estudio EPICARDIAN fueron seleccionadas a través del padrón oficial de población aportado por el Servicio de Estadística del Ayuntamiento de Madrid al 31 de Marzo de 1991. Según este, en el Barrio de Lista había ins- critos 5.540 habitantes mayores de 64 años, en el Barrio de Las Margaritas 2.251 según la misma fuente ( $15,5 \%$ de sus habitantes) y 2.228 habitantes en la zona básica de salud de Arévalo según padrón municipal. Los criterios para elegir a un sujeto fueron que tuviera 65 años o más y residiera más de 6 meses en el lugar seleccionado. En Margaritas y Arévalo fueron seleccionados para el estudio todos los sujetos incluidos en el censo, sin embargo en Lista se seleccionó una muestra aleatoria debido al gran número de individuos mayores de 65 años residentes en la zona. Concretamente, se estratificó la población por segmentos etarios y sexo, y se seleccionó al 30\% de los sujetos incluidos en cada estrato.

La estimación del tamaño muestral en este tipo de estudios se basa fundamentalmente en asegurar la potencia suficiente que garantice la aparición de los principales eventos cardiovasculares objeto de estudio, que en el estudio al que nos referimos fue de 6.395 sujetos, que otorgaban un poder estadístico superior al $80 \%$ para detectar casos nuevos de CI o ACV en el estudio prospectivo, asumiendo una tasa de incidencia acumulada del $20 \%$ en tres años.

Los estudios de cohortes se inician habitualmente con un estudio transversal que persigue 3 objetivos fundamentales: por un lado la exclusión de aquellos sujetos con discapacidades y minusvalías relevantes (según clasificación de minusvalías, deficiencias y discapacidades del INE); pacientes con enfermedades terminales en el momento de estudio con dificultades y bajas probabilidades de supervivencia (p.e. pacientes en tratamiento quimioterápico o radioterápico por cáncer) y pacientes ingresados en residencias de la tercera edad $\mathrm{u}$ hospitales de larga estancia en el momento del examen inicial. Por otra parte, el estudio transversal permite identificar los casos prevalentes de enfermedad bajo estudio con el objetivo de seleccionar la población libre de enfermedad (a riesgo), así como clasificar a esta población a 
riesgo según diferentes niveles de exposición a los factores de riesgo.

En el estudio EPICARDIAN el trabajo se desarrolló en dos fases. En la primera se realizó el reclutamiento y recogida de datos de los participantes mediante entrevista domiciliaria realizada por enfermeras entrenadas y certificadas en las técnicas y mediciones realizadas. La segunda fue de selección de posibles casos positivos en base a los resultados de los cuestionarios iniciales, y de búsqueda y recopilación de toda la información clínica por dos médicos debidamente entrenados.

La entrevista fue estructurada y recogía datos demográficos como edad, sexo, estado civil, nivel de estudios, profesión habitual e información socio-económica, un cuestionario estandarizado y validado a partir del proyecto OMS-MONICA sobre antecedentes personales y familiares de hipertensión arterial (HTA), diabetes, hipercolesterolemia, tabaquismo, consumo de alcohol, de fármacos, y cambios recientes de peso, así como un cuestionario sobre acontecimientos cardiovasculares y cerebrovasculares padecidos, adaptado también del cuestionario del proyecto MONICA (Monitoring Trends and Determinants in Cardiovascular Disease $)^{14}$.
La entrevista incluía también el cuestionario sobre dolor torácico y disnea de Rose ${ }^{17,18}$.

A los sujetos «no respondedores» a la fase domiciliaria se les envió por correo un cuestionario autoadministrado, y/o se contactó con familiares, convivientes, vecinos, o médicos de Atención Primaria, con el objetivo de obtener información clave sobre su salud general, factores de riesgo y enfermedades cardiovasculares en particular. Igualmente se solicitó periódicamente información al INE sobre causa, fecha y lugar de la muerte de los individuos de la cohorte fallecidos.

Con los resultados de la fase domiciliaria los médicos del estudio seleccionaron a los individuos que habían respondido afirmativamente a las preguntas sobre enfermedad cardiaca, angina y por tanto candidatos a ser examinados posteriormente en la $2^{\mathrm{a}}$ fase del estudio. El procedimiento empleado fue la utilización del «método de búsqueda en frío» utilizado en el proyecto MONICA ${ }^{17}$ modificado en los proyectos REGICOR e IBERICA $^{19}$ (figura 1).

En esta fase se tuvo acceso a todos los informes clínicos, historias hospitalarias y de atención primaria que fueron necesarios

Figura 1

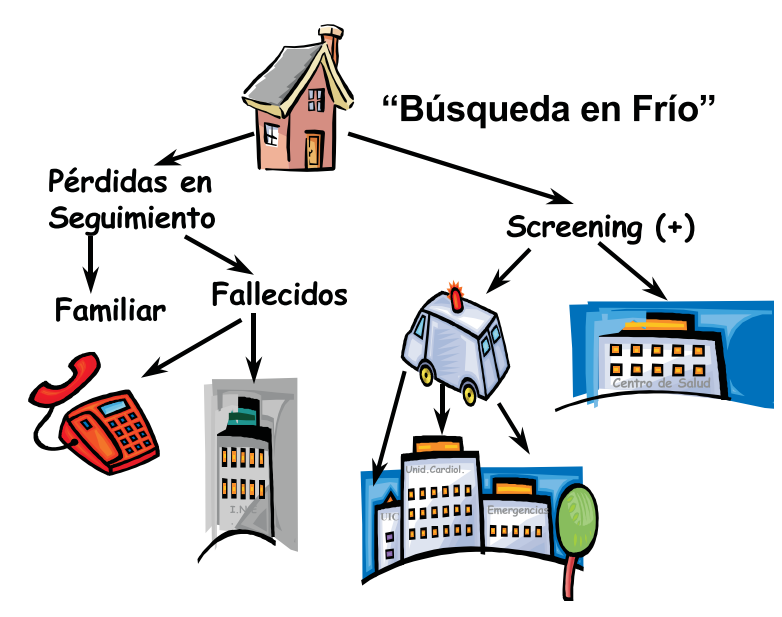


para la confirmación del evento cardiovascular. Igualmente se solicitó periódicamente información al INE sobre causa, fecha y lugar de la muerte de los individuos de la cohorte fallecidos.

Como en cualquier otro diseño de investigación, en un estudio de cohortes es fundamental definir estrictamente las variables de estudio que, en el caso que nos ocupa, exigió un gran esfuerzo al equipo investigador.

Así para la definición de angina se utilizó el cuestionario de Rose, adaptado y validado para su empleo en la población española ${ }^{20} \mathrm{y}$ se consideró angina segura si los participantes respondían afirmativamente a las tres primeras preguntas sobre dolor torácico y se detenían, notando alivio o desapareciendo éste en 10 minutos o menos. Para la definición de IAM se realizó una búsqueda de criterios clínicos, electrocardiográficos y enzimáticos compatibles. Se clasificó como IAM seguro aquél que presentó ECG definitivo o síntomas típicos o atípicos mal descritos, junto con ECG probable y enzimas anormales, o aquél con síntomas típicos, enzimas anormales y ECG isquémico. Se consideró IAM posible o mиerte de origen coronaria en aquellos sujetos vivos con síntomas típicos en los que los enzimas y el ECG no permitían clasificarlos en la categoría de definitivo, sin que hubiera evidencia de otro diagnostico, o aquellos pacientes fallecidos cuando no existía una evidencia clara de otra causa de muerte. Por último, se consideró $N o$ $I A M$, en pacientes vivos, cuando la combinación de síntomas y pruebas diagnosticas no permitió clasificar al IAM en la categoría de seguro ni se presentaron síntomas típicos para poder clasificarlos en la categoría de posible, o cuando el episodio pudo ser explicado por otros diagnósticos. En los casos fatales se consideró no IAM cuando se trató de una muerte que tenía otro diagnóstico clínico o necrópsico como causa.

Para el diagnostico de ictus, se consideraron positivos aquellos sujetos que respon- dieron afirmativamente o de forma dudosa a las preguntas de cribado y que fueron citados posteriormente por un neurólogo. El neurólogo evaluaba y clasificaba al sujeto en 3 categorías: no patología cerebrovascular, si el sujeto no tenía criterios de diagnóstico o no era posible excluir otro proceso, enfermedad cerebrovascular confirmada o enfermedad cerebrovascular dudosa si el sujeto cumplía criterios pero no se encontró documentación relevante en los informes médicos existentes.

Todos los instrumentos utilizados en ambas fases fueron validados mediante un estudio en una muestra de la población incluida. En el caso de IAM se seleccionaron 935 individuos a los que se les realizó un ECG de 12 derivaciones. 176 individuos resultaron positivos tanto para las preguntas de screening como para el ECG. Los sujetos con ondas Q en el ECG y con ausencia de síntomas, se clasificaron como falsos negativos de IAM (n:33). 132 sujetos resultaron falsos positivos (sujetos que respondieron positivamente al cuestionario y que en el ECG no se pudo confirmar el IAM. Por último, 594 sujetos fueron negativos tanto para el cuestionario como para el ECG. Estos datos permitieron confirmar que la sensibilidad del sistema de screening era del $84 \%$ y la especificidad del $81 \%$.

La exactitud del screening de ictus fue evaluada previamente en dos estudios epidemiológicos ${ }^{16,17}$. La primera pregunta del cuestionario alcanzó una sensibilidad del $94,5 \%$ y una especificidad del $95,7 \%$. El uso combinado de las cuatro preguntas del cuestionario obtuvo una sensibilidad del 99,2\% y una especificidad del 91,1\%. Nuestro estudio identificó 257 casos de ictus, de los que 187 fueron positivos para las preguntas de screening. La existencia de falsos negativos fue investigada mediante la selección aleatoria de 350 individuos entre los sujetos con screening negativo, en los que se identificaron tres casos adicionales. Por el contrario, no se encontraron casos falsos positivos. Por 
lo tanto, la sensibilidad final del instrumento (ajustada por falsos negativos) fue del $72 \%$ y la especificidad del $100 \%$.

La definición de las variables independientes es también fundamental para la seguridad de los resultados, así la evaluación de los diferentes FRCV en el estudio EPICARDIAN se realizó a través del cuestionario estandarizado administrado por enfermeras entrenadas. Además se recogieron medidas objetivas de los factores de riesgo: peso y talla, cálculo del índice de masa corporal y cintura/cadera. Para el cálculo de la presión arterial media se realizaron 3 diferentes lecturas de PA ( 2 sentadas y una de pie) tras 1 minuto de reposo y con un esfigmomanómetro de mercurio con un manguito adecuado al diámetro del brazo del sujeto. Todas las medidas fueron realizadas por enfermeras debidamente entrenadas ${ }^{22}$.

También se realizó una analítica sanguínea con medidores capilares portátiles de colesterol total y glucosa (Lipotrend@ para colesterol total y Glucometer@ para glucosa capilar) ${ }^{23}$. Se revisaron también datos sobre el uso de medicación para cualquiera de los FRCV durante la entrevista.

En los estudios de cohortes la determinación del evento a estudio obliga al seguimiento de toda la cohorte, durante un periodo de tiempo que depende fundamentalmente de la duración del periodo de latencia de la enfermedad a estudio.

Así el estudio se inició en 1994, con un estudio transversal, que se repitió en 1998, y que en el momento actual está repitiéndose, por lo que se estima un seguimiento medio de la cohorte de 9 años.

Además del tiempo de seguimiento es fundamental poder completar el seguimiento en la mayoría de los sujetos incluidos inicialmente en el estudio.

En este estudio y con el objeto de garantizar al máximo el conocimiento de la distri- bución del evento entre los sujetos expuestos y no expuestos, el proceso de seguimiento de la cohorte se adaptó a la situación de cada zona. En Arévalo los médicos participantes contactaron con los pacientes o sus familiares periódicamente y registraron cualquier cambio de domicilio, institucionalización o defunción en el momento que ocurrían. En Getafe y Lista se solicitó periódicamente información al INE sobre las defunciones de los miembros de la cohorte y se establecieron contactos telefónicos con los sujetos de la cohorte o sus allegados. Durante 1998, se realizó un segundo estudio transversal y se pusieron en marcha los mismos mecanismos de contacto y método de entrevista que en 1994. En los sujetos que no pudieron ser localizados en el momento de la entrevista, se buscó igualmente información sobre su estatus vital en el INE y sobre posibles acontecimientos cardiovasculares a través de los servicios de urgencias, los hospitales de referencia, y los centros de salud de la zona.

Para el análisis de los datos se utilizaron los estadísticos básicos de centralización (media aritmética con su desviación estándar y distribución cuartiles) y para describir las variables continuas y de dispersión valores máximos y mínimos y el recorrido. Para variables categóricas se utilizó la distribución de frecuencias relativas (prevalencia) con los intervalos de confianza al $95 \%{ }^{24}$.

Para la comparación de proporciones se utilizó la prueba $\chi 2$ de Pearson y la $\chi 2$ de tendencia lineal para variables cualitativas con varias categorías ordenadas. Para la comparación de medias la prueba de $\mathrm{t}$ de Student-Fisher en el caso de variables independientes binarias y el Análisis de la Varianza si se trataban de variables de más de dos categorías. Se consideró como estadísticamente significativo cualquier valor de $\mathrm{p}<0,05$. El método de análisis actuarial Kaplan-Meier ${ }^{25}$ se utilizó para calcular la supervivencia de la cohorte y estimar las tasas de incidencia. Se realizó un calculo de la incidencia acumulada y se estudia el ries- 


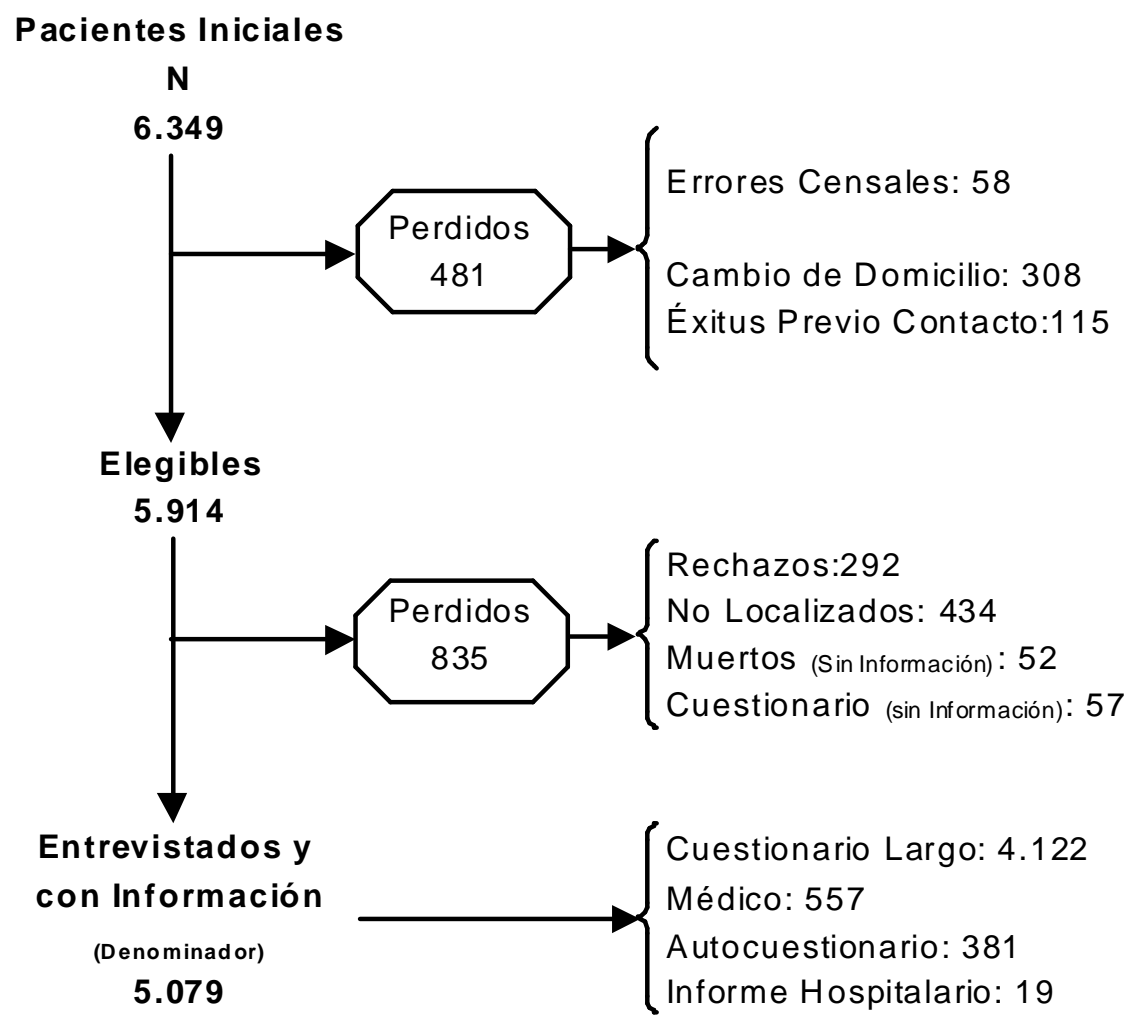

go relativo de los diferentes factores de riesgo relacionados con la enfermedad de estudio.

\section{RESULTADOS}

El número de sujetos elegibles en las tres zonas del estudio en 1994 fue de 6.395. En 481 casos $(7,5 \%)$ no se obtuvo respuesta alguna debido a errores censales, cambios de domicilio o éxitus previo a la fecha de inicio del estudio. 778 individuos $(12,1 \%)$ rechazaron o no pudieron ser localizados tras varios intentos. En 57 sujetos no se pudo completar el cuestionario de estudio. En total, se consiguió entrevistar a 5.079 sujetos (tasa global de participación 85\%) (figura 2). Se compararon las características sociodemográficas tales como edad, sexo, nivel cultural y lugar de residencia y clínicas, PAS y PAD, glucemia, frecuencia cardiaca, colesterol total, IMC e índice cintura/cadera, de los sujetos que rechazaron y no se encontraron diferencias significativas entre los grupos.

La muestra final estaba compuesta por un $42,3 \%$ de hombres y un $57,7 \%$ de mujeres. El 90,6\% eran menores de 84 años. La edad media de la cohorte al inicio del estudio fue de 74,17 (+- 6,89) años, 76 en mujeres y 73 en hombres (tabla 1) La distribución de la 
Tabla 1

Distribución de las características de las personas de la muestra

\begin{tabular}{|c|c|c|c|}
\hline & Hombres (\%) & Mujeres (\%) & Total \\
\hline \multirow{2}{*}{$65-74$} & 1.324 & 1.655 & 2.979 \\
\cline { 2 - 4 } & $(26,1)$ & $(32,6)$ & $(58,7)$ \\
\hline \multirow{2}{*}{$75-84$} & 650 & 972 & 1.622 \\
\cline { 2 - 4 } & $(12,8)$ & $(19,1)$ & $(31,9)$ \\
\hline \multirow{2}{*}{$85+$} & 176 & 302 & 478 \\
\cline { 2 - 4 } & $(3,5)$ & $(5,9)$ & $(9,4)$ \\
\hline \multirow{2}{*}{ Total } & 2.150 & 2.929 & 5.079 \\
\cline { 2 - 4 } & $(42,3)$ & $(57,7)$ & $(100,0)$ \\
\hline
\end{tabular}

muestra por centros fue de 1.541 sujetos en Lista (30,3\%), 1.788 en Arévalo $(35,2 \%)$ y $1.750(34,5 \%)$ en Getafe.

De los 5.079 sujetos elegidos 290 (5,7\%) habían padecido un IAM previo al 1 de Mayo de 1994 (día de prevalencia) por lo que la población a riesgo y libre de IAM al inicio del seguimiento era de 4.769 sujetos. Durante el periodo de seguimiento no fue posible localizar a 393 sujetos $(8,2 \%)$ y rechazaron participar de nuevo en la segunda encuesta 147 (3\%). En 119 (2,5\%) el cuestionario resultó incompleto. En todos los individuos que habían fallecidos durante el seguimiento se obtuvo información sobre la causa de muerte, bien a través del INE, de informes hospitalarios, historias de atención primaria, o a partir de la información de familiares. El porcentaje de participación en el estudio de incidencia fue del 85,32\% (4.069 sujetos), El tiempo medio de seguimiento de los sujetos de la cohorte fue de 3,22 años con un rango de 0,1 a 4,83 años (desviación típica de 1,13 años). No existieron diferencias significativas entre centros, grupos de edad y sexo en el tiempo de seguimiento.

537 sujetos $(10,6 \%)$ reconocieron haber padecido un episodio agudo cardiovascular o haber padecido dolor torácico de más de 30 minutos de duración. Tras la comprobación de cada uno de los acontecimientos referidos por los pacientes, mediante la metodología de investigación mencionada, se pudieron confirmar 201 infartos seguros y hasta 315 si se suman los IAM clasificados como probables. Esto indica una prevalencia global de IAM seguro en la muestra de un $4 \%$ (201/5.079) (IC 95\%: 3,4-4,5), y de IAM seguro más probables del 6,2\% (IC 95\%: 5,5-6,9). La prevalencia de IAM seguro fue más elevada en los varones. 6,7\% (IC 95\%:5,63-7,79) que en las mujeres, $2 \%$ (IC $95 \%: 1,51-2,55)(\mathrm{p}<0,001)$. Y la diferencia se mantuvo al analizar IAM seguro más probable (varones: 9,1\% (IC 95\%:7,9-10,4) y mujeres: 4,1\% (IC 95\%: 3,4-4,8), (p<0,001). La edad media de los sujetos que habían padecido un IAM fue de 74,38+/-6 años, y una prevalencia ligeramente mayor entre los 
pacientes de mayor edad, sin que se pudieran demostrar diferencias significativas. (PA 0.293). Sin embargo al analizarlo conjuntamente con el IAM probable se mostró que las mujeres más añosas tenían una prevalencia mayor de IAM que las más jóvenes. $[3,6 \%(2,75-4,6 \%)$ en el grupo de 65 a 75 años y $6,80 \%(4,28-10,3) \mathrm{p}<0,001]$. La prevalencia de IAM seguro es mayor entre los varones que en las mujeres para cualquier grupo de edad y en éstas la prevalencia aumenta mucho en los estratos de mayor edad. No se encontraron diferencias significativas entre las distintas áreas de estudio.

La prevalencia de los diferentes factores de riesgo basándose en la entrevista fueron $1.830(45,2 \%)$ para hipertensión según criterios de la OMS y $2.676(43,9 \%)$ basados en la contestación del cuestionario. Para hipercolesterolemia las tasas fueron del $26,4 \%$ segun criterios del NCP y del $29 \%$ según el cuestionario. Para diabetes el $13,4 \%$ de los sujetos fueron positivos según criterios de la OMS y 15,6\% según el cuestionario. 582 sujetos $(11,3 \%)$ eran fumadores.

La incidencia acumulada en 3,2 años fue de 1,4\% (IC 95\%: 1,1-1,8) de IAM seguro no fatal, de 1,1\%(IC95\%:0,74-1,37) de IAM posible, 1,17 (IC95\%: 0,824-1,48) de IAM seguro fatal o muerte por IAM, y $1,13 \%$ (IC 95\%: 0,824-1,48) de muerte súbita.

\section{DISCUSIÓN}

Los estudios de cohortes son uno de los modelos de investigación en epidemiología cardiovascular que mejor permiten conocer las características, patrón de ocurrencia y los factores relacionados con la cardiopatía isquémica en una población determinada y su análisis e interpretación correctas permiten aplicar con cierta confianza sus resultados a los sujetos representados en el mismo. Ésta fue la razón primordial, que decidió este tipo de estudio a la hora de estimar la prevalencia e incidencia de cardiopatía isquémica (angina e IAM) en la población anciana en España.

Este ambicioso objetivo obliga a realizar un diseño, ejecución y análisis minucioso de cada una de sus fases. Más concretamente obliga a realizar una selección adecuada no sesgada de sujetos libres de enfermedad al inicio del seguimiento, a que éste sea lo suficientemente prolongado y exhaustivo, a que se midan correctamente las variables de interés, y a que los procedimientos de análisis controlen la acción de otras variables que puedan explicar los hallazgos (factores de confusión $)^{26}$.

Que el IAM y el ictus, aun siendo eventos puntuales en el tiempo, sean consistentes, nos ha permitido, infiriendo este dato como la comprobación evidente de la enfermedad cardiovascular en los sujetos estudiados, evaluar la prevalencia de cardiopatía isquémica e ictus ${ }^{27}$. Así un sujeto que desarrolló un evento hace años, ha sido incluido como prevalente en nuestro estudio. Somos conscientes, no obstante, que esta inferencia plantea una limitación en este tipo de estudios, con una tendencia clara a la infraestimación de los resultados.

En esta cohorte se realizó de forma muy cuidadosa la inclusión de los participantes al inicio del estudio. A partir de la población empadronada, y mediante muestreo aleatorio estratificado por edad y sexo, se intentó contactar con 6.395 sujetos, de los que sólo a un $12,1 \%$ no se consiguió localizar o se negaron a participar. Esta alta tasa de participación se consiguió mediante una persecución extrema de aquellos sujetos de más difícil contacto, implicando a los familiares y médicos de cabecera, facilitándole al sujeto la entrevista, que era domiciliaria, e intentando contactar con el sujeto hasta 6 veces antes de clasificar al individuo elegido como no localizado.

Aunque las tasas de rechazo en el estudio fueron pequeñas, se realizó un análisis com- 
parativo de las características demográficas de los sujetos participantes y no respondedores, no pudiéndose demostrar diferencias significativas entre ambos grupos.

Por todo lo anterior, se podría inferir que los 5.079 sujetos incluidos en el estudio representan adecuadamente a los ancianos del barrio de Salamanca de Madrid, de Getafe y de Arévalo y que los resultados pueden probablemente generalizarse a la población anciana de estas regiones españolas.

Con el fin de garantizar la calidad de los datos en el estudio prospectivo, de los 5.079 sujetos incluidos en el estudio transversal y entendiendo que el objetivo último era la búsqueda de los sujetos libres de enfermedad, durante la evaluación de cada caso, en todos ellos se sobrestimó la presencia de evento, eliminándose para el seguimiento tanto los casos de evento seguro como los posibles, para evitar al máximo el sesgo de selección protoptópico (presencia de enfermedad subclínica).

Otro problema añadido a este tipo de estudios basados en cuestionarios es el sesgo de recuerdo, ya que la determinación del evento se basa principalmente en el recuerdo del sujeto $^{28}$ en este caso se puso un empeño especial en realizar una pregunta clave amplia, que determinó un valor predictivo negativo muy alto.

Una de las mayores dificultades que aparecen en un estudio de cohortes, principalmente en ancianos, es el seguimiento de la misma. Se establece así, de forma teórica, un $30 \%$ de perdidas como límite para asegurar la validez del estudio.

Con el fin de minimizar las perdidas en la mayoría de los estudios de cohortes se suelen establecer múltiples estrategias. En nuestro estudio se realizó una evaluación mediante cuestionario al inicio y al final del seguimiento, como recomiendan alguno de los estudios epidemiológicos a nivel interna- cional más importantes ${ }^{28}$. Además se mantuvo contacto con los sujetos de la cohorte, bien telefónico o bien a través de sus médicos de cabecera anualmente, y se vigiló con periodicidad anual el registro.

Ha habido, no obstante, cierto número de perdidas inevitables, alcanzando una tasa de participación a lo largo de los 3 primeros años del estudio de un $85 \%$, comparables a las de otros estudios de cohortes en patología cardiovascular. El estudio de Framingham, por ejemplo, tuvo un $69 \%$ de respuesta ${ }^{29}$, y el estudio REGICOR, realizado en nuestro país, consiguió una tasa de respuesta del $72 \%{ }^{30}$.

Sólo en cohortes cerradas (no poblacionales), como en el estudio de los trabajadores de la factoría Pirelli de Manresa, Tomás Abadal et al pudieron alcanzar tasas de participación que superaron el $90 \%$ a los 20 años de seguimiento ${ }^{31}$.

Por último, con el objeto de conocer si la ocurrencia de enfermedad cardiovascular era comparable en los sujetos que completaron el estudio y en los que no se pudo completar el seguimiento, se realizó un análisis adicional que demostró que en los sujetos que se perdieron $(14,8 \%)$ la distribución de sus factores de riesgo cardiovascular y características socio-demográficas al inicio del estudio no difiere de los sujetos estudiados completamente ${ }^{32,33}$. Sólo se encontró una ligera proporción mayor de varones y de pacientes con demencia entre los no respondedores, lo que es consistente con otros estudios similares ${ }^{34}$.

La muestra de estudio elegida es, sociodemográficamente hablando, comparable a la población de referencia, con un porcentaje, no significativamente mayor, de mujeres y de ancianos del medio rural. El número de sujetos mayor de 80 años incluidos fue importante $(22,4 \%)$, y la edad media de la cohorte alcanzó los 74 años (65-103 años), aspecto que aporta un gran interés a este estudio, ya que existen pocos datos sobre este subgrupo poblacional. 
La distribución de los distintos factores de riesgo en nuestra población, es característica de una población occidental, mediterránea, anciana, con una alta prevalencia de los distintos $\mathrm{FRCV}^{35}$.

Cuando se compara nuestro estudio con otros que intentan caracterizar el riesgo cardiovascular en la población se aprecian bastantes similitudes ${ }^{36,37}$, con la salvedad de que la mayor parte de estos estudios se basan en poblaciones más jóvenes ${ }^{38,39}$.

En resumen, las características de los ancianos incluidos en nuestro estudio corresponde a una población con elevada prevalencia de factores de riego cardiovascular, que parece estar en consonancia con los datos que aportan los escasos estudios en ancianos en nuestro país ${ }^{40}$. En las mujeres el perfil de riesgo fue significativamente peor que en los varones, lo que sin más puede indicar una selección natural con una mayor mortalidad a edades más precoces en el sexo masculino, lo que se debe tener en cuenta a la hora de valorar las tasas de prevalencia e incidencia del IAM.

Por último, la comparación de las tres poblaciones incluidas ha permitido un acercamiento a las diferencias entre regiones, ya que la diferencia entre distintas poblaciones es un fenómeno universal recogido en múltiples estudios ${ }^{41}$. Esta variabilidad puede deberse a numerosos factores, genéticos, ambientales, diferencias sociales y en ocasiones a las diferentes técnicas de medición. En este estudio los factores referentes a la metodología empleada no interfieren en los resultados, pues se han unificado criterios y estandarizado mediciones de forma similar en las tres poblaciones. Este propósito ha justificado el esfuerzo realizado a la hora de analizar cada uno de los resultados por población de origen de los sujetos.

Consideramos por tanto que un estudio de cohortes es la mejor manera de aproximarse a la incidencia de una enfermedad y a la valoración de determinados comportamientos o factores que influyen en su aparición. En el estudio EPICARDIAN se ha pretendido evaluar con esta metodología la incidencia, prevalencia y factores de riesgo de la enfermedad cardiovascular.

\section{BIBLIOGRAFIA}

1. McKeon T, Lowe CR. Introducción a la medicina social. México: Siglo XXI; 1986.

2. Dodu SRA. Emergence of cardiovascular diseases in developing countries. Cardiology 1988; 75:5664.

3. Uemura K, Pisa Z. Trends in cardiovascular disease mortality in industrialized countries since 1950 . Wld Hlth Statis Quart 1988; 41: 155-78.

4. Rosamond WD, Chambless L.E., Folsom AR, Cooper LS, Conwill DE, Clegg L, Wang C-H, Heiss G, Trends in the incidence of myocardial infarction and in mortality due to coronary heart disease, 1987 to 1994. N Engl J Med 1998; 339:861-7.

5. Banegas JR, Rodríguez F, Villar F, Rey J. Perspectiva epidemiológica del riesgo cardiovascular en España. Iberoamerican Journal of hypertension 1996, 1(suppl 2): 40-5.

6. Instituto Nacional de Estadística. Defunciones según la Causa de Muerte. Años 1975-1998. Madrid: INE; 1979-2001.

7. Gabriel Sánchez R. Hacia un estudio epidemiológico sobre factores de riesgo cardiovascular en el anciano en España. Rev Esp Geriatr y Gerontol $1990 ; 25,6: 383-8$

8. Dawber TR. The Framingham Study. The epidemiology of atheroesclerotic disease. Cambridge, Mass: Harvard University Press; 1980.

9. The Pooling Project Research Group. Relationship of blood pressure, serum colesterol, smoking habit, relative weight and ECG abnormalities to incidence of major coronary events. Final report of the Pooling Project. J Chronic Dis. 1978; 31:201-306.

10. Keys A. Seven Countries. A multivariate análisis of death and coronary Heart disease. Cambridge, Mass: Harvard University Press; 1980

11. Fried LP, Borhani NO, Enright P, et al. The Cardiovascular Health Study: design and rationale. Ann Epidemiol 1991;1:263-276. 
12. Yano K, Reed DM, McGee DL: Ten year incidence of coronary heart disease in the Honolulu Heart Program: relationship to biological and lifestyle characteristics. Am J Epidemiol 1984;119:653666 .

13. Tomás Abadal L, Balaguer Vintró I, Bernat E. Factores de riesgo e incidencia de nuevos casos en el estudio prospectivo de la cardiopatía isquémica de Manresa. Rev Esp Cardiol 1976; 29: 127-35

14. Tomàs i Abadal L, Varas Lorenzo C, Pérez I, Puig T, I Balaguer Vintró I. Factores de riesgo y morbimortalidad coronaria a los 28 años de seguimiento de una cohorte con baja incidencia de la enfermedad: el estudio de Manresa. Rev Esp Salud Pública 2004; 78:

15. Gabriel R, Alonso M, Bermejo F, Muñiz J, López I, Suárez C, et al. Proyecto Epicardian: Estudio epidemiológico sobre enfermedades y factores de riesgo cardiovasculares en ancianos españoles. Diseño, método y resultados preliminares. Rev Esp Geriatr Gerontol 1996:31 (6): 327-34.

16. Hennekens ChH, Buring JE. Epidemiology in medicine. Boston: Little, Brown and Co; 1987.

17. World Health Organization Cardiovascular Diseases Unit: WHO MONICA Project: MONICA Manual. Geneva: World Health Organization; 1990.

18. Rose GA, Blackburn H, Gillyn RF, Prineas RJ. Métodos de encuesta sobre enfermedades cardiovasculares (2nd ed.) Ginebra: Organización Mundial de la salud; 1982

19. Elosua R, Fiol M, Tormo MJ, Segura A, Bregada J, Villegas M, Ayensa A, Marrugat J en nombre del grupo IBERICA. Letalidad poblacional del infarto agudo de miocardio en cuatro regiones españolas. Estudio IBERICA. Rev Esp Cardiol 1999.

20. Cosín J, Asín E, Marrugat J, Elosua R, Aros F, De los Reyes $\mathrm{M}$ et al, for the PANES study group. Prevalence of angina pectoris in Spain. Eur J Epidemiol 1999; 15:323-30.

21. Tunstall-Pedoe H, Kuulasmaa K, Amouyel P, Arveiler D, Rajakangas AM, Pajak A. Myocardial infarction and coronary deaths in the World Health Organization MONICA Project. Registration procedures, event rates, and case-fatality rates in 38 populations from 21 countries in four continents. Circulation. 1994 Jul;90(1):583-612.

22. Curb JD, Labarthe DR, Cooper SP, Cutter GR, Hawkins CM. Training and certification of blood pressure observers. Hypertension 1983;5:610-4.
23. National Cholesterol Education Program. Recommendations for Improving Cholesterol Measurements. Bethesda, MD; US. Department of Health and Human Services. Public Health Service. National Institutes of Health. National Heart, Lung, and Blood Institute. February 1990; NIH. Pub. No. 902964.

24. Statistics without tears. A Primer for Non-mathematicians. Derek Rowntree. London: Penguin Books; 1981.

25. Kaplan EL, Meier P. Non parametric estimation from incomplete observations. J Am Statist Ass $1958 ; 53: 457-81$

26. Fletcher RH, Fletcher SW, y Wagner EH. Epidemiología Clínica. Barcelona: Ediciones Consulta; 1989.p. 75-89.

27. Gabriel Sánchez R y Gómez de la Cámara A. Métodos de investigación en cardiología clínica. (I). Tipos de diseño básicos. Estudios observacionales en cardiología clínica (I). Rev Esp Cardiol 1996;49:837-51

28. Tuomilehto J, Kuulasmaa K. WHO MONICA Project: assessing CHD mortality and morbidity. Int J Epidemiol. 1989;18 (3 Suppl 1):S38-45.

29. Dawber TR, Kannel WB, Lyell LP. An approach to longitudinal studies in a community: the Framingham Study. Ann NY Acad Sci 1963;107:539-56.

30. Perez G, Pena A, Sala J, Roset P, Masia R, Marrugat $\mathrm{J}$ ant the REGICOR Investigators. Acute myocardial infarction case fatality, incidence and mortality rates in a population registry in Gerona, Spain, 1990-1992. Int J Epidemiol 1998;27:599-604.

31. Estudios observacionales: historia natural y pronostico en cardiología en Métodos de investigación en cardiología clínica Varas C, Tomás Abadal L. Editores F. Fernández Avilés; J Alonso Martín; J Pérez Villacastín. Madrid: Sociedad Española de Cardiologia; 1998.

32. Joseph H, Abramson MB. Prevention of cardiovascular disease in the elderly. Public Health Rev $1985 ; 13: 165-223$.

33. Abramson JH, ed. Métodos de estudio en Medicina Comunitaria. Madrid: Díaz de Santos; 1990.p.132133 y p.153-157.

34. Dewhurst G, Wood DA, Walker F, Lampe FC, Jeffreys M, Cooper M and Williams JD. A population survey of cardiovascular disease in elderly people: design, Methods and Prevalence results. Age Ageing 1991;20:353-60.

Rev Esp Salud Pública 2004, Vol. 78, N. ${ }^{\circ} 2$ 
35. Sáez T, Suárez C, Blanco F y Gabriel R. Epidemiología de las enfermedades cardiovasculares en la población anciana española Rev Esp Cardiol 1998; 51: 864-873.

36. Ettinger WH, Wahl PW, Kuller LH. Lipoproteins lipids in older people. Results from the Cardiovascular Health Study. Circulation 1992; 86:858-69.

37. Corti MCh, Guralnik JM, Salive ME et al HDL Cholesterol Predicts Coronary Heart Disease Mortality in Older Persons. JAMA 1995; 274: 539-544)

38. Masiá R, Pena A, Marrugat J, Sala J, Vila J. Pavesi M, Covas M, Aubó C, Elosua R and the Regicor Investigators. High prevalence of cardiovascular risk factors in Gerona, Spain, a province with low myocardial infarction incidence. J Epidemiol Community Health 1998;52:707-15.
39. Kannel WB, Cobb J Framingham Study: risk factors in the cohort aged 65 and older. En: Lewis B, Mancini M, Farinaro E, editores. Prevention of cardiovascular heart disease in the elderly. Londres: Current Medical Literature; 1991.p. 3-18.

40. Trenkwalder P, Ruland D, Stender M et al Prevalencia, Consciencia, Tratamiento y Control de la HTA en una población de edad superior a los 65 años: resultados del estudio de Starnberg sobre epidemiología del parkinsonismo y de la HTA en el anciano (STEPHY). J Hypertens 1994; 1: 405412

41. Alonso M, Rodríguez Artalejo F, Del Rey Calero J. Relationship between socioeconomic status and ischaemic heart disease in cohort and case-control studies: 1960-1993. Int J Epidemiol1997;27:35058. 